\title{
Performance of Space Time Block Codes and Quasi Orthogonal Space Time Codes on Fading Channel
}

\author{
Sajjad Ahmed Ghauri ${ }^{1}$, Bilal Arshad ${ }^{1}$, Agha Saif $^{1}$, I. M. Qureshi ${ }^{2}$ \\ ${ }^{1}$ National University of Modern Languages, Islamabad, Pakistan \\ ${ }^{2}$ Air University, Islamabad, Pakistan
}

\begin{abstract}
In this paper the performance of Space Time Codes (STC) \& Quasi Orthogonal Space Time Block Codes (QOSTBC) has been analyzed. Use of multiple antennas is an emerging approach for recovering fading and reducing the bit error rate. Space diversity is introduced in space time codes. In Alamouti Space Time Code (STC) two transmit antennas are used that provide relatively better results than no coding approach. Alamouti uses a code matrix at transmitter and at receiver end it uses MLD and estimates the output signals. Space time block codes are with arbitrary number of antennas. It uses a huge code matrix at encoder and decodes using MLD. QOSTBC is one of the latest variants of the STBCs. The encoded symbols are mapped in the transmission matrix which is decoded at the receiver end using pair wise maximum likelihood decoding (MLD). Pair wise MLD is the specialty of the QOSTBC. The performance of STBC is far better than Alamouti and has no match with no coding. The performance comparison of QOSTBC, Alamouti, STBC and no coding is showed using simulations in MATLAB. Space time codes works with the price of redundancy bits and extra antenna price. Simulation results for QOSTBC also shows better performance with the price of slightly increased decoding complexity.
\end{abstract}

\section{Introduction}

Mobility, accessibility and probability are the factors that make a wireless system more attractive to the end user. Today the world is merging into wireless technology and despite of the fact that wired system is more reliable, more stable and have a better performance, the user are trying to get into the regime of wireless technology.

Fading leads to attenuation of the signal. The fading causes the system to be limited to a certain bit rate and quality of service. There is more chance of a high error performance which is not attractive to end user. The fading in the channel is caused due to certain factors present in the free air those factors might be temperature, scattering, diffraction, attenuation or non LOS communication.

One of the major drawbacks of these wireless systems is the limited bandwidth available to them. This of course does not concern the user but is indeed a big issue. Having a limited bandwidth means that only a limited number of users can be entertained in the available spectrum.

In order to increase the capacity of the wireless systems for providing high bit-rates to the users, new techniques have been developed over the years. One of the techniques is using space time block codes. And its variant QOSTBC is a type of non-orthogonal space time block codes and gives better performance when the system is composed of more than two antennas.

\section{System Model}

(a) Alamouti STC

One of the first and basic STCs is Alamouti Space Time Code (ASTC) that provides transmits diversity. Using 2 transmit antennas instead of one is the main theme behind the space time codes. Inserting the transmit diversity decreases the error rate considerably.

At the receiver end one and two antennas are used. An information source is taken that may be a stream of bits or a voice. BPSK modulation is done. The encoder takes an input of 2 symbols from the modulated output. The encoder has an Alamouti matrix that maps those bits according to following matrix X:

$$
X=\left[\begin{array}{cc}
x_{1} & -x_{2}{ }^{*} \\
x_{2} & x_{1}{ }^{*}
\end{array}\right]
$$

In first time slot $x_{1}$ and $x_{2}$ will be sent and on 2nd time slot $\mathrm{x}_{2}{ }^{*}$ and $\mathrm{x}_{1}{ }^{*}$ would be transmitted. The transmitted signals pass through Rayleigh fading channel. Rayleigh faded noise is been added to them in that channel. The received signals are represented 
by $r_{1}$ and $r_{2}$. The following equation shows the received signals at the receiver.

$$
r_{i}=\sum_{\mathrm{i}=1}^{\mathrm{n}_{T}} a_{i} * x_{i}+n_{i}
$$

where

$\mathrm{n}_{\mathrm{T}}=$ Number of transmit antennas

$\mathrm{r}_{\mathrm{i}}=$ Received bits from ith transmit antenna

$\mathrm{a}_{\mathrm{i}}=$ The path gain matrix

$\mathrm{x}_{\mathrm{i}}=$ The bits at the $\mathrm{i}^{\mathrm{th}}$ transmit antenna

$\mathrm{n}_{\mathrm{i}}=$ Rayleigh faded noise for $\mathrm{i}^{\mathrm{th}}$ antenna

At the receiver end maximum likelihood decoding is used. We can implement MLD linearly in ASTC decoder. MLD forms decision variables and estimates the received signal $r_{i}$. Following is the formulae for the decision variables

$$
R_{i}=\sum_{\mathrm{t}=1}^{\mathrm{n}_{T}} \sum_{\mathrm{j}=1}^{\mathrm{n}_{\mathrm{R}}} r_{t} * a_{j} * \delta_{t}
$$

$\mathrm{R}_{\mathrm{i}}=$ decision variable

$\mathrm{r}_{\mathrm{t}}=$ Received signal of the $t^{\text {th }}$ transmit antenna.

$\delta_{\mathrm{t}}=$ Sign of the transmitted bit

After finding the decision variables through MLD the receiver estimates from the constellation that satisfies the following equation.

$$
s_{i}=\arg \min \left(\left|R_{i}-s\right|^{2}+\left(-1+\sum_{k, l}\left|\alpha_{k, l}\right|^{2}\right)|s|^{2}\right)
$$

\section{(b) Space Time Block Codes}

As discussed in ASTC that provided diversity for two transmit and one, two receive antenna. Alamouti scheme was proposed for two transmit antennas, to increase the range of transmit antennas to an arbitrary number Space Time Block Codes STBC were introduced. STBC is actually a generalized form of ASTC in which we can increase the transmit antennas keeping in touch with the spatiality of ASTC of orthogonality between the transmitted signals on respected antennas. In this section STBC will be discussed and we will also focus on factors like code rate because with increase in antennas there comes complexity in the code matrix and we might have to sacrifice some bandwidth for it. At the encoder side information bits are produced and modulated using BPSK modulation scheme. The code matrix for the encoder of STBC is a bit complex. For real signals the code matrix $\mathrm{X}$ shown below is for 4 transmit antennas, it will be different for other numbers of antennas.

$$
X=\left\{\begin{array}{cccc}
x_{1} & -x_{2} & -x_{3} & -x_{4} \\
x_{2} & x_{1} & x_{4} & -x_{3} \\
x_{3} & -x_{4} & x_{1} & x_{2} \\
x_{4} & x_{3} & -x_{2} & x_{1}
\end{array}\right\}
$$

where the code rate is $\mathrm{R}=\mathrm{k} / \mathrm{p}, \mathrm{k}$ is number of bits taken by the encoder and $\mathrm{p}$ is number of bits transmitted through each antenna in a single time slot. The code matrix $\mathrm{X}$ for real signals the code rate is 1 . Code rate of 1 means that there would be no need for increasing the bandwidth. If the code rate is less than 1 then the bandwidth would have to be expanded by the factor $1 / \mathrm{R}$. The encoder takes $\mathrm{k}=4$ real modulated symbols $\mathrm{x}_{1}, \mathrm{x}_{2}, \mathrm{x}_{3}, \mathrm{x}_{4}$ as its input. Then these symbols are passed through the code matrix and they are mapped all over it. Then output is a $4 \times 4$ matrix. The matrix is then mounted on each antenna row by row and those rows are then transmitted. In first time slot $\mathrm{x}_{1}, \mathrm{x}_{2}, \mathrm{x}_{3}, \mathrm{x}_{4}$ are transmitted from Tx1. The transmitted signals pass through Rayleigh fading channel. The received signals are represented by $r_{1}$ and $r_{2}$. At the receiver end maximum likelihood decoding is used. We can implement MLD linearly in STBC decoder. MLD forms decision variables and estimates the received signal $r_{i}$.

\section{(c) Quasi Orthogonal Space Time Block Codes}

QOSTBC is a type of non-orthogonal STBC. Its transmission matrix is not entirely non orthogonal but is partially orthogonal. It is more suitable when using more than two antennas at the transmitter side in the wireless system. QOSTBC provide full diversity, rate one and simple pair wise decoding can be performed and relatively better results are achieved. Four transmit antennas and one receive antenna are used. The information source provides the bits to be transmitted. Two types of modulation are used: QPSK and rotated QPSK. In rotated QPSK, the symbols are rotated and form a rotated constellation. Rotated QPSK provide full diversity in QOSTBC. The symbols to be transmitted are fed to the encoder which than maps them in the following general matrix $\mathrm{X}$

$$
\mathrm{X}=\left[\begin{array}{cccc}
x_{1} & x_{2} & x_{3} & x_{4} \\
-x_{2}{ }^{*} & x_{1}{ }^{*} & -x_{4}{ }^{*} & x_{3}{ }^{*} \\
-x_{3}{ }^{*} & -x_{4}{ }^{*} & x_{1}{ }^{*} & x_{2}{ }^{*} \\
x_{4} & -x_{3} & -x_{2} & x_{1}
\end{array}\right]
$$

We have four transmit antennas and each antenna will transmit one element from a row in one timeslot hence one row of symbols will be transmitted in one timeslot. So in the first timeslot, $\mathrm{x}_{1}, \mathrm{x}_{2}, \mathrm{x}_{3}$ and $\mathrm{x}_{4}$ will be transmitted and vice versa. Let the symbols to be 
transmitted are $s_{1}, s_{2}, s_{3}$ and $s_{4}$. The symbols which rotate are $s_{3}$ and $s_{4}$ to get full diversity. Then the rotated symbols in radians are $s_{3}=e^{j \varphi s_{3}}$ and $s_{4}{ }^{\prime}=e^{j \varphi s_{4}}$. The other two symbols $s_{1}$ and $s_{2}$ will be transmitted without rotation. These transmitted symbols passed through a Rayleigh fading channel after passing through the Rayleigh fading channel, the faded symbols are received at the receiver antenna. For QOSTBC, we use pair wise maximum likelihood decoding. We can decode a pair of symbols independently in QOSTBC. We can decode the received symbols using the following equations:

For $\mathrm{s}_{1}$ and $\mathrm{s}_{4}$

$$
\begin{aligned}
f_{14}\left(s_{1}, s_{4}\right)= & \sum_{m=1}^{M}\left[\left(\left|s_{1}\right|^{2}+\left|s_{4}\right|^{2}\right)\left(\sum_{n=1}^{4}\left|\alpha_{n, m}\right|^{2}\right)\right. \\
& +2 \Re\left\{\left(-\alpha_{1, m} r_{1, m}^{*}-\alpha_{2, m}^{*} r_{2, m}-\alpha_{3, m}^{*} r_{3, m}-\alpha_{4, m} r_{4,}^{*}\right.\right. \\
& +\left(-\alpha_{4, m} r_{1, m}^{*}+\alpha_{3, m}^{*} r_{2, m}+\alpha_{2, m}^{*} r_{3, m}-\alpha_{1, m} r_{4, m}^{*}\right) s_{c} \\
& \left.+4 \Re\left\{\alpha_{1, m} \alpha_{4, m}^{*}-\alpha_{2, m}^{*} \alpha_{3, m}\right\} \Re\left\{s_{1} s_{4}^{*}\right\}\right],
\end{aligned}
$$

For $s_{2}$ and $s_{3}$,

$$
\begin{aligned}
f_{23}\left(s_{2}, s_{3}\right)= & \sum_{m=1}^{M}\left[\left(\left|s_{2}\right|^{2}+\left|s_{3}\right|^{2}\right)\left(\sum_{n=1}^{4}\left|\alpha_{n, m}\right|^{2}\right)\right. \\
& +2 \Re\left\{\left(-\alpha_{2, m} r_{1, m}^{*}+\alpha_{1, m}^{*} r_{2, m}-\alpha_{4, m}^{*} r_{3, m}+\alpha_{3, m} r_{4,}^{*}\right.\right. \\
& +\left(-\alpha_{3, m} r_{1, m}^{*}-\alpha_{4, m}^{*} r_{2, m}+\alpha_{1, m}^{*} r_{3, m}+\alpha_{2, m} r_{4, m}^{*}\right) s_{3} \\
& \left.+4 \Re\left\{\alpha_{2, m} \alpha_{3, m}^{*}-\alpha_{1, m}^{*} \alpha_{4, m}\right\} \Re\left\{s_{2} s_{3}^{*}\right\}\right]
\end{aligned}
$$

where $s_{1} \& s_{2}=$ transmitted symbol and $\alpha$ are path matrix and $\mathrm{r}$ is symbol matrix.

\section{Simulation Results}

The implementation of ASTC for two transmit, one receive and two transmit, two receive antenna has been done.

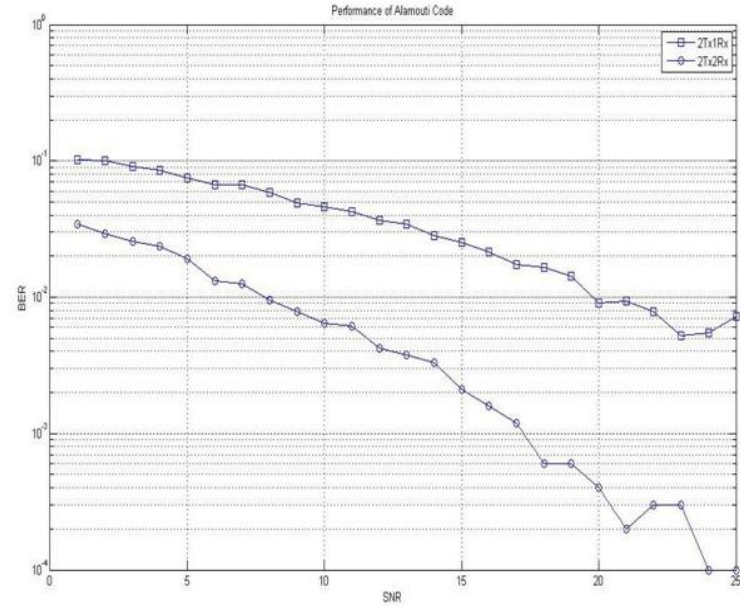

(a)

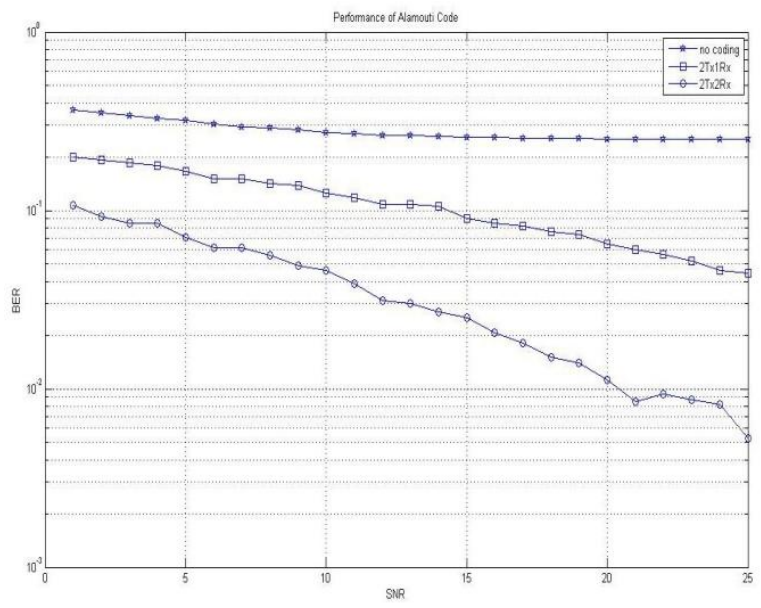

(b)

Figure 1: (a) Performance Comparison of Alamouti with one and two receive antennas with 2 transmit antennas, (b) Comparison of Alamouti with transmission without coding. 


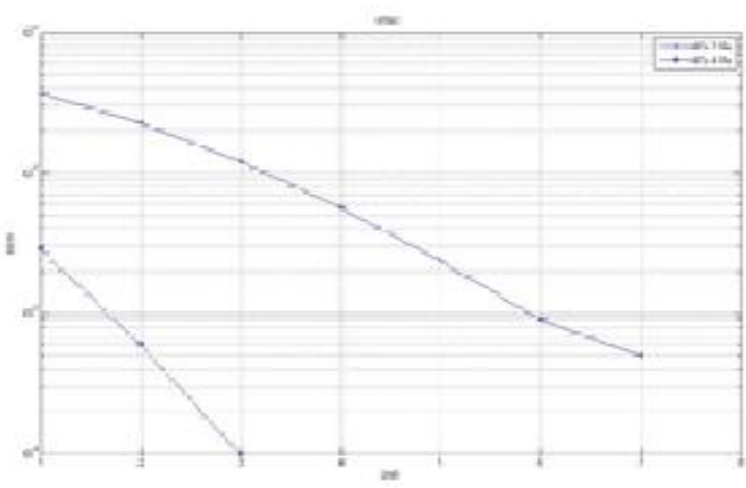

(a)

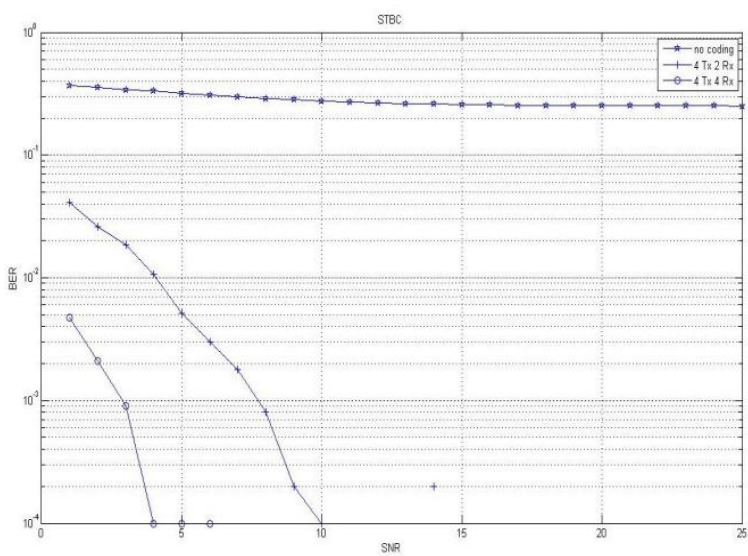

(b)

Figure 2: (a) STBC with 2 and 4 receive antennas and 4 transmit antennas. (b) Comparison of STBC with transmission without coding.

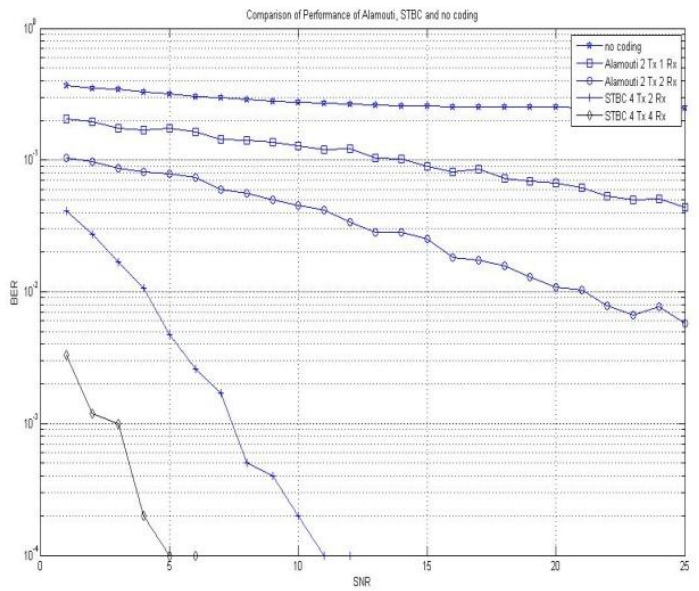

Figure 3: Simulation Result for Alamouti, STBC and no coding.
The simulation result of Figure 4 shows the performance comparison of QOSTBC with no coding and BER performance is much better with low SNR.

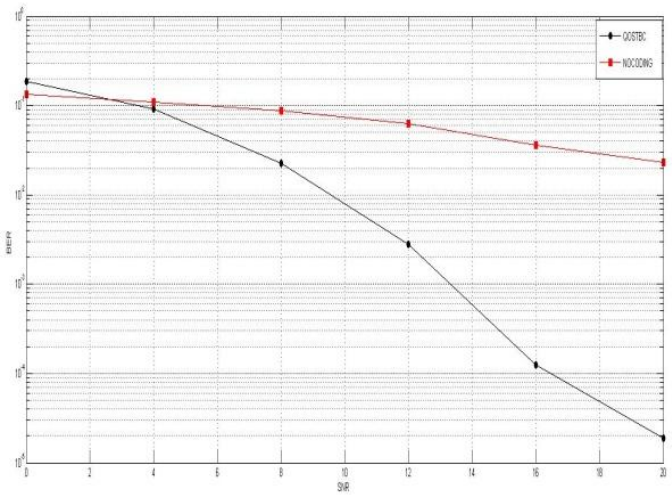

Figure 4: QOSTBC with 1 receive antennas and 4 transmit antennas compared with no coding on Rayleigh fading channel

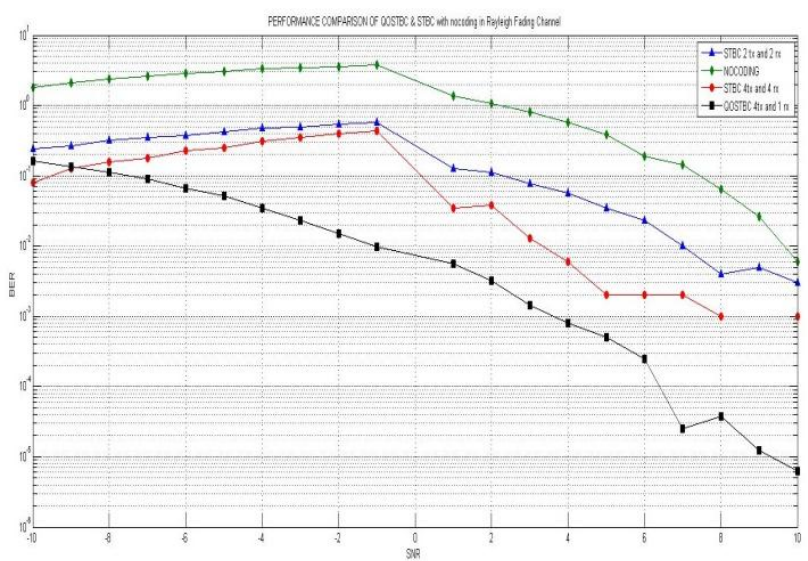

Figure 5: QOSTBC with 1 receive antennas and 4 transmit antennas compared with no coding and STBC with 4 and 2 transmit and receive antennas on Rayleigh fading channel.

The performance comparison of QOSTBC and STBC on Rayleigh fading channel is shown in Figure 5. The simulation shows that QOSTBC performs better at low SNR but high complexity.

\section{Conclusion}

The simulation result illustrate that out of Alamouti and STBC, STBC are worth to be implemented on the wireless communication systems. As they are providing far less bit error rate as compare to no coding communication. Increase in 
the number of transmit and receive antennas enhances the performance of STBC.

The simulation Figure 3 shows the comparison of Alamouti, STBC and no coding. STBC with 4 transmit and 4 receive antennas has the best result of all. So we can say that if we increase the number of antennas the result would be better but the tradeoff in such approach is the increase in the computations at the receiver end.

The QOSTBC can provide full code rate and full diversity. Its decoding complexity is a little higher than the orthogonal STBCs but is still less than that of the non-orthogonal STBCs. QOSTBC outperforms (in a bit-error rate sense) the fully orthogonal 4antenna STBC over a good range of signal-to-noise ratios (SNRs). It performs better at higher SNRs and better than STBC.

\section{References}

[1]B. Vucetic, J. Yuan, "Space-Time Coding", John Wiley \& Sons, England, 2003.

[2] Z. Liu, G. B. Giannakis, S. Zhou, B. Muquet, "Spacetime coding for boradband wireless communications", Wireless Communications and Moblie Computing, vol. 1, no. 1. pp. 35-53, Jan. 2001.

[3]Sajjad Ahmed Ghauri, M. Sajid Javed, M. Raza Parvez ,Bilal Ahmed "Performance of Space Time Codes on Fading Channel" IEEE conference of Information Society UK, 2012.

[4]M. T. Ivrlac, T. P. Kurpjuhn, C. Brunner, W. Utschick, "Efficient use of fading correlation in MIMO systems", 54th VTC, Atlantic City, USA, vol. 4, pp. 2763-2767, Oct. 2001.

[5] J. G. Proakis, Digital Communications, 4th Ed., McGraw-Hill, New York, 2001.

[6]Sajjad Ahmed Ghauri, M. Ehsan ul Haq, Farhan Sohail, Bilal Ahmad "Performance of Space Time Trellis Code on Fading Channel" 8th IEEE conference on Wireless \& Mobile computing, Networking \& communication, Spain, 2012.

[7]S. M. Alamouti, "A simple transmit diversity technique for wireless communications", IEEE Journal Select. Areas Commun., vol. 16, no. 8, pp. 1451-1458, Oct. 1998.

[8]A. Naguib, V. Tarokh, N. Seshadri and A.Calderband, "A space=time coding modem for high data rates wireless communications".

[9]S. M. Alamouti,"A simple transmit diversity technique for wireless communications".
[10]V. Tarokh, H. Jafarkhani and A.R.Calderband,"Spacetime block codes from orthogonal designs".

[11]J. Grimm, M.P.Fitz andJ.V.Krogmeier, "Further results in space-time coding for Rayleigh fading".

[12]A.R.Hammons and H.E. Gammal,"On the theory of space-time codes for PSK modulation”.

[13]S. Baro, G. Bauch and A. Hansmann, "Improved Codes for space-time trellis coded modulation".

[14]Q. Yan and R.S.Blum, "Optimum space-time convolutional codes".

[15]Z. Chen, J.Yuan and B.Vucetic, "Improved space-time trellis coded modulation scheme on slow Rayleigh Fading channels".

[16]Z. Chen, J.Yuan, B.Vucetic and K. Lo, "space-time trellis coded modulation with three and four transmit antennas on slow fading channels".

[17]Z. Chen, J.Yuan, B.Vucetic and W. Firmanto,"Performance analysis and design of space-time coding on fading channel". 